Telomere length of background vs pa1AT hepatocytes

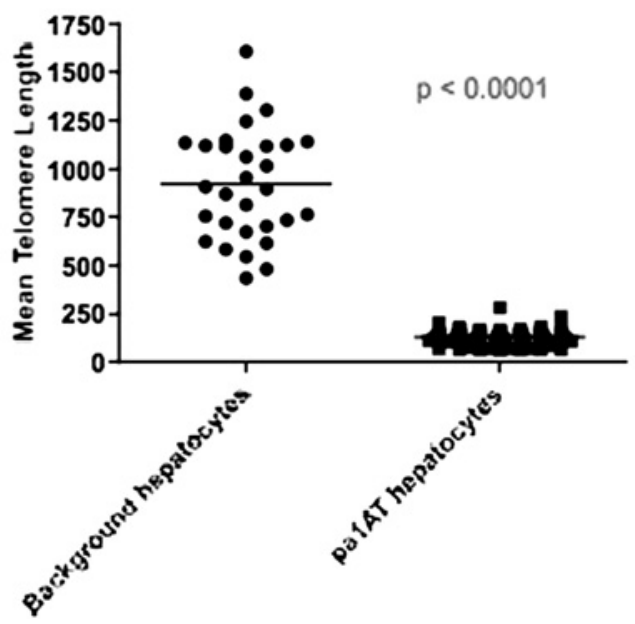

Abstract P90 Figure 2 Hepatocyte telomere length in patients with a1AT deficiency with or without polymerised a1AT.

Conclusion Senescence, characterised by increased nuclear size and shortened telomeres, was present in hepatocytes from patients with both homozygous and heterozygous a1AT related liver disease. The novel 2C1 antibody showed that these markers of senescence were even more marked in cells expressing pa1AT. The steps between pa1AT expression and accelerated senescence are a clear target for therapeutic intervention.

\section{P91 DUAL MECHANISM OF NK CELL INHIBITION BY THE HCV CORE 35-44 PEPTIDE}

doi:10.1136/gutjnl-2011-300857a.91

K S Cheent, S I Khakoo. Department of Hepatology, St Mary's Campus, Imperial College London

Introduction Disease association studies have shown that the inhibitory killer cell immunoglobulin receptor KIR2DL3 and its HLA-C1 allotype ligands are protective against chronic HCV infection. A number of studies have shown increased expression of another inhibitory natural killer (NK) cell receptor, CD94:NKG2A,

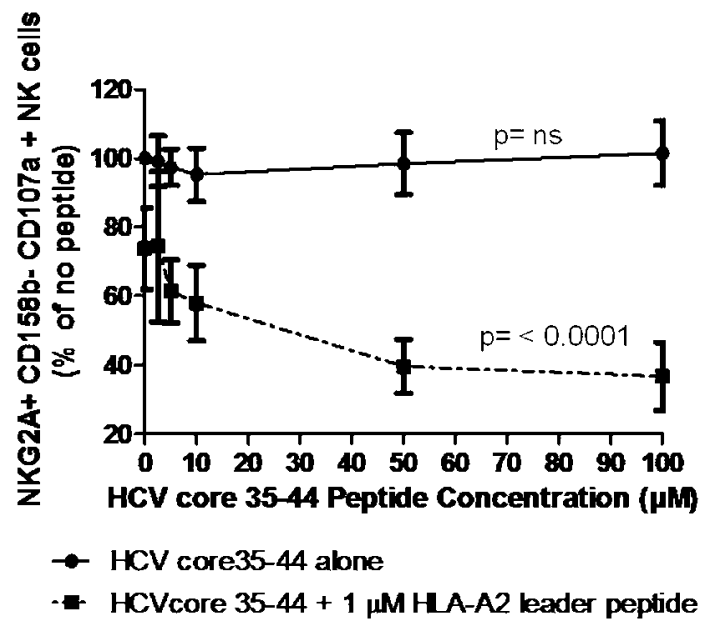

Abstract P91 Figure 1 HCVcore $35-44$ induced inhibition of KIR2DL2/3 (CD158b) + NK cells.

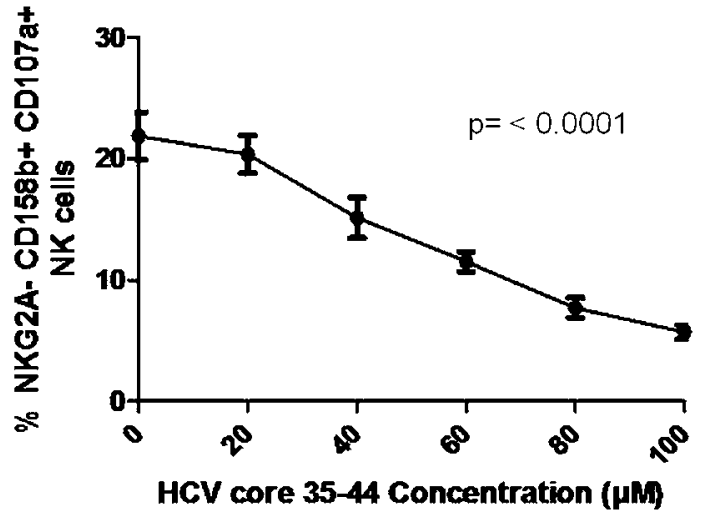

Abstract P91 Figure 2 HCV core 35-44 alone does not inhibit via CD94:NKG2A.

in chronic HCV infection. The ligand for this receptor, HLA-E, usually binds to leader peptides derived from other HLA class I molecules. Up-regulation of HLA-E with concomitant inhibition of NK cells has been thought to be induced by peptides derived from several viruses including one derived from HCV (HCV core $_{35-44}$ ) which thus represents a mechanism of immune escape.

Method To determine the effects of viral peptides on NK cell inhibition, we pulsed the TAP-deficient 721.174 cell line with host and virus derived peptides and used these as target cells in flow cytometry assays of NK cell degranulation (measured by CD107a expression).

Results In functional experiments HCV core $_{35-44}$ inhibited degranulation of the total NK cell population. Unexpectedly, this was due to inhibition of KIR2DL2/3-positive NK cells (one way ANOVA $p<0.0001)$, but not NKG2A-positive NK cells $(p=0.94)$. However, HCV core $_{35-44}$ peptide increased cell surface expression of both HLA-C and HLA-E. To rationalise this with the previous findings we performed peptide mix experiments using the natural peptide ligands for HLA-E. Such peptides, derived from the leader sequence of host HLA class I molecules, efficiently inhibited NKG2A-positive NK cells. In the presence of a low concentration of a host HLA class I leader sequence peptide, HCV core $_{35-44}$ had a synergistic effect in suppressing NK cell activity $(p<0.0001)$. A synergistic effect at CD94:NKG2A was also demonstrable for peptides derived from EBV and HIV viruses, which in isolation did not inhibit NK cells, implying that this is a general mechanism for NK cell inhibition.

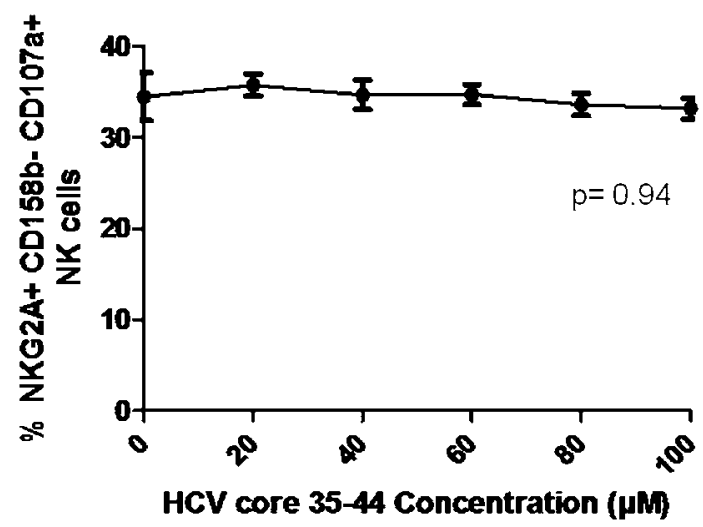

Abstract P91 Figure 3 However, in the presence of a host HLA (HLAA2) derived leader peptide, HCV 35-44 induces synergistic inhibition at CD94:NKG2A. 
Conclusion HCV core $_{35-44}$ inhibits NK cell activity via two distinct mechanisms, directly via KIR2DL2/3, and synergistically via the CD94:NKG2A receptor. This synergistic interaction at CD94:NKG2A represents a novel mechanism for inhibiting NK cells. It demonstrates the sensitivity of NK cells to small changes in the peptide content of HLA class I, and is thus potentially a sensitive mechanism for viral escape from the host innate immune response.

\section{P92 CD161 $+\gamma-\delta$ T CELLS: DEFINING THEIR ROLE IN PATIENTS WITH AND WITHOUT CHRONIC HEPATITIS C}

doi:10.1136/gutjnl-2011-300857a.92

${ }^{1} \mathrm{~N}$ Rajoriya, ${ }^{1} \mathrm{C}$ Willberg, ${ }^{2} \mathrm{~B}$ Seigal, ${ }^{1} Y$ uHoi Kang, ${ }^{3} \mathrm{~J}$ Phillips-Hughes, ${ }^{4} \mathrm{~J}$ Collier, ${ }^{1} \mathrm{E}$ Barnes, ${ }^{2} \mathrm{R}$ Thimme, ${ }^{2} \mathrm{P}$ Klenerman. ${ }^{1}$ Peter Medawar Building for Pathogen Research, Oxford University; ${ }^{2}$ Department of Medicine, University of Freiburg; ${ }^{3}$ Radiology Department, Churchill Hospital, Oxford; ${ }^{4}$ Department of Gastroenterology, John Radcliffe Hospital, Oxford

Introduction $\gamma-\delta$ T cells have been found in blood/liver of patients infected with hepatitis C (HCV). CD161+ is a C-type lectin found on the surface of T cells in humans. HCV specific T-cells have been found to express CD161 with enrichment within the liver. The role \& function of CD161 $+\gamma-\delta$ cells has not been established in HCV. Aim To explore the phenotype and function of CD161+ $\gamma-\delta$ T cells in humans, and to assess the impact on these cells of HCV infection. Method Whole blood/Peripheral Blood Mononuclear cell (PBMC) antibody staining with subsequent flow cytometry was performed to assess phenotype. Function was examined by Intracellular Cytokine staining (ICS). Intrahepatic lymhocytes (IHLs) were isolated from patients having liver biopsies for clinical indications with paired blood samples. Cord blood samples were used after ethical approval gained and consent from mothers.

Results In healthy controls, the CD161 subset encompassed a mean of $82.1( \pm 12.8) \%$ of $\gamma-\delta$ cells. The CD161 subset expressed more CXCR3/CCR6/IL-18R ( $p=0.03 / 0.01$ and 0.03 respectively) than the CD161- subset. The CD161 + cells expressed significantly more If-y/ TNF- $\alpha$ ( $p=0.001$ respectively) than the negative subset, and more Granzyme A, B and K and perforin in keeping with a Th1 profile. $20 \%$ of $\gamma-\delta$ cells from cord blood samples expressed CD161, suggesting it is an innate feature but expanded throughout life. Whole blood $\gamma-\delta$ populations were significantly reduced in HCV compared to healthy donors $(70 \% \vee 40 \%, p=0.0031)$, with the proportion of CD161 $+\gamma-\delta$ T-cells reduced in chronic HCV $(82.1 \%$ vs $39 \%, p=0.006)$. The $\gamma-\delta$ cells in HCV expressed significantly more activation markers (CD38/CD69) and CXCR6 than in healthy controls irrespective of CD161 status. Of the CD161+ $\gamma-\delta$ cells, the predominant subset were $\mathrm{V} \delta 2$ in healthy controls blood however in HCV this was significantly reduced $(p=0.0173)$ with the V $\delta 1$ subset dominating. On isolation of IHLs $(n=17: 8 \mathrm{HCV}, 3 \mathrm{HBV}$ and rest NAFLD/NASH), there was an enrichment of CD3 $+\gamma-\delta$ T-cells in liver tissue compared to blood irrespective of CD161 status. On subanalysis there were no statistical differences between CD161 status of the $\gamma-\delta$ cell T-cells when comparing viral v non-viral aetiologies. Conclusion Our data suggests that HCV infection does directly reduce the $\gamma-\delta$ T-cell population in peripheral blood: mainly the CD161 subset. The virus also causes a reversal in the CD161 $\gamma-\delta$ T-cell population from $\mathrm{V} \delta 2$ to $\mathrm{V} \delta 1$ in the periphery, however it appears the enrichment of $\gamma-\delta \mathrm{T}$ cells to the liver is not specific to CD161 + cells or to HCV infection. The CD161 phenotype present at birth but expanded throughout life. The CD161 subset appears to display a more Th1 profile and unlike their CD8 CD161++ $\alpha-\beta$ counterparts, CD161 expression by $\gamma-\delta$ T-cells is not tightly linked to a Type-17 differentiation pathway.

\section{P93 NFKB ACTIVATION BY TLR AGONISTS IS DEFICIENT IN MACROPHAGES LACKING STAT2}

doi:10.1136/gutjnl-2011-300857a.93

${ }^{1} \mathrm{~W}$ Alazawi, ${ }^{2} \mathrm{~A}$ Gamero, ${ }^{1} \mathrm{H}$ Heath, ${ }^{3} \mathrm{P}$ Smith, ${ }^{4} \mathrm{M}$ Jacobs, ${ }^{1} \mathrm{~J}$ Waters, ${ }^{1} \mathrm{G}$ R Foster ${ }^{1}$ Barts \& The London School of Medicine; ${ }^{2}$ Temple University, Philadelphia, USA; ${ }^{3}$ St George's University London; ${ }^{4}$ University College London

Introduction Engagement of Toll-like receptors (TLRs) is an important initiator of the innate immune response in inflammatory liver diseases and leads to release of a range of inflammatory cytokines. TLRs signal through divergent signal transduction pathways but the majority lead to activation of members of the NFKB family. STAT2 is an essential component of canonical signalling through the type I and type III interferon (IFN) receptor but, unlike other members of the Stat family, is not believed to be involved in other signal transduction pathways.

Aim We examined the role of STAT2 on inflammatory signalling.

Results Loss of STAT2 markedly reduced immortalised macrophages' response to a range of TLR agonists (lipopolysaccharide (LPS), poly I:C and IL-1)-production of TNF $\alpha$ and RANTES proteins and a range of inflammatory mRNAs were decreased. The reduction in inflammatory cytokine production could be reversed by reconstitution with STAT2 but blockade of type I IFN signalling did not reproduce the phenotype. Restoration of the normal response to LPS could be achieved with tyrosine phosphorylation defective STAT2 indicating that STAT2 interacts with these signalling pathways without phosphorylation on Tyr690. The multiplicity of STAT2's effect suggests a common defect to these signalling pathways. There were no abnormalities in the activation of early signalling components in the absence of STAT2, however, levels of phosphorylated $I \kappa \mathrm{B}$, although not total $I \kappa \mathrm{B}$, were reduced in STAT2 $^{-/-}$cells. Phosphorylation of this inhibitor of NFKB leads to its degradation and release of NFKB proteins into the nucleus. We found that translocation of the NFKB protein p 65 into the nucleus and its subsequent binding to DNA was impaired in STAT2 deficient cells.

Conclusion These data suggest that STAT2 is a critical component of the TLR signalling response to early inflammatory stimuli, in particular through maintaining the normal phosphorylation of $I \kappa \mathrm{B}$.

\section{P94 RAPAMYCIN HELPS MAINTAIN THE REGULATORY PHENOTYPE OF CYTOCHROME P450IID6-SPECIFIC TREG EXPANDED FROM PATIENTS WITH AUTOIMIMUNE HEPATITIS TYPE 2 BY REDUCING THE NUMBER OF IFN $\gamma^{+}$ CELLS}

doi:10.1136/gutjnl-2011-300857a.94

B Holder, C R Grant, Y Ma, G Mieli-Vergani, D Vergani, M S Longhi. Institute of Liver Studies, King's College London School of Medicine at King's College Hospital, London, UK

Introduction Control of $\mathrm{T}$ cell reactivity to cytochrome P450IID6 (CYP2D6) is key to immune-tolerance restoration in autoimmune hepatitis type 2 (AIH-2). $\mathrm{CD} 4{ }^{+} \mathrm{CD} 25^{+}$regulatory $\mathrm{T}$ cells (Treg) central to autoreactive $T$ cell regulation, are impaired in AIH-2. Cell therapy based on CYP2D6-specific Treg (CYP-Treg) could provide specific control over effectors of liver damage in AIH-2. We have generated CYP-Treg from AIH-2 patients and demonstrated that these cells exert greater suppression than polyclonal Treg. Whether CYP-Treg can undergo expansion maintaining their functional phenotype is untested.

Aim To assess CYP-Treg functional phenotype over 2-week expansion in AIH-2 patients. 